ARTICLE

DOI: $10.1038 /$ s41467-018-05712-5

\title{
Biology and genome of a newly discovered sibling species of Caenorhabditis elegans
}

Natsumi Kanzaki (10 1,8, Isheng J. Tsai (1) 2, Ryusei Tanaka ${ }^{3}$, Vicky L. Hunt ${ }^{3}$, Dang Liu (1) 2, Kenji Tsuyama (i) ${ }^{4}$, Yasunobu Maeda ${ }^{3}$, Satoshi Namai ${ }^{4}$, Ryohei Kumagai ${ }^{4}$, Alan Tracey ${ }^{5}$, Nancy Holroyd ${ }^{5}$, Stephen R. Doyle (1) ${ }^{5}$, Gavin C. Woodruff ${ }^{1,9}$, Kazunori Murase ${ }^{3}$, Hiromi Kitazume ${ }^{3}$, Cynthia Chai $^{6}$, Allison Akagi ${ }^{6}$, Oishika Panda (1) ${ }^{7}$, Huei-Mien Ke ${ }^{2}$, Frank C. Schroeder ${ }^{7}$, John Wang ${ }^{2}$, Matthew Berriman (1) ${ }^{5}$, Paul W. Sternberg (1) ${ }^{6}$, Asako Sugimoto (i] ${ }^{4} \&$ Taisei Kikuchi (i) ${ }^{3}$

A 'sibling' species of the model organism Caenorhabditis elegans has long been sought for use in comparative analyses that would enable deep evolutionary interpretations of biological phenomena. Here, we describe the first sibling species of $C$. elegans, C. inopinata n. sp., isolated from fig syconia in Okinawa, Japan. We investigate the morphology, developmental processes and behaviour of $C$. inopinata, which differ significantly from those of $C$. elegans. The 123-Mb C. inopinata genome was sequenced and assembled into six nuclear chromosomes, allowing delineation of Caenorhabditis genome evolution and revealing unique characteristics, such as highly expanded transposable elements that might have contributed to the genome evolution of $C$. inopinata. In addition, $C$. inopinata exhibits massive gene losses in chemoreceptor gene families, which could be correlated with its limited habitat area. We have developed genetic and molecular techniques for $C$. inopinata; thus $C$. inopinata provides an exciting new platform for comparative evolutionary studies.

\footnotetext{
${ }^{1}$ Forestry and Forest Products Research Institute, Tsukuba 305-8687, Japan. ${ }^{2}$ Biodiversity Research Center, Academia Sinica, Taipei city 11529, Taiwan. ${ }^{3}$ Faculty of Medicine, University of Miyazaki, Miyazaki 889-1692, Japan. ${ }^{4}$ Laboratory of Developmental Dynamics, Graduate School of Life Sciences, Tohoku University, Sendai 980-8577, Japan. ${ }^{5}$ Wellcome Trust Sanger Institute, Wellcome Genome Campus, Hinxton CB10 1SA, UK. ${ }^{6}$ HHMI and Division of Biology and Biological Engineering, Caltech, Pasadena, CA 91125, USA. ${ }^{7}$ Boyce Thompson Institute, and Department of Chemistry and Chemical Biology, Cornell University, Ithaca, NY 14853, USA. 8 Present address: Kansai Research Center, Forestry and Forest Products Research Institute, 68 Nagaikyutaroh,

Momoyama, Fushimi, Kyoto 612-0855, Japan. ${ }^{9}$ Present address: Department of Biology, Institute of Ecology and Evolution, University of Oregon, Eugene, OR 97403-5289, USA. These authors contributed equally: Natsumi Kanzaki, Isheng J. Tsai, Ryusei Tanaka, Vicky L. Hunt. Correspondence and requests for materials should be addressed to A.S. (email: asugimoto@m.tohoku.ac.jp) or to T.K. (email: taisei_kikuchi@med.miyazaki-u.ac.jp)
} 
C aenorhabditis elegans, a free-living nematode inhabiting organic matter-rich environments like rotting fruits and stems in many parts of the world, is one of the most intensively used model organisms in biological studies. Key features of this animal include it's short and prolific life cycle, small body size, genetic manipulability, fully described developmental programme, and well-characterised genome. Commonalities of the genetic pathways in C. elegans with other organisms including humans led to seminal discoveries in a wide variety of research fields, including neuroscience, development, signal transduction, cell death, ageing, and small RNAs ${ }^{1}$. One of the few missing pieces of $C$. elegans to be an exemplary model organism has been its sibling species, which would further enhance C. elegans' role as a model organism by allowing evolutionary interpretations of biological phenomena through deep comparisons between species that have more recently diverged. C. briggsae is currently the most widely used satellite model, but at the level of DNA sequence, this species is as divergent from C. elegans as humans are from mice ${ }^{2}$. Other species, including C. brenneri and C. remanei, compose a clade with $C$. briggsae in the Elegans group but C. elegans remains phylogenetically distant ${ }^{2}$. Here, we report a discovery of the longsought sibling species of C. elegans, Caenorhabditis inopinata n. sp. in Ishigaki Island, Okinawa, Japan ("inopinata" meaning surprising or unexpected in Latin as the biological and phylogenetic characters of this new species were a big surprise for the authors). Although known habitats of most other Caenorhabditis species are soil, rotting organic materials, and leaf-litter environments $^{3}$, C. inopinata was isolated from fresh syconia of the fig Ficus septica and is likely to have a close phoretic association with the fig wasp Ceratosolen bisulcatus.

\section{Results and discussion}

Taxonomic description. Order Rhabditida Chitwood, 1933

Family Rhabditidae Örley, 1880

Genus Caenorhabditis Osche, 1952

Caenorhabditis inopinata n. sp.

More detailed taxonomic description of the new species is provided as the Supplementary note 1 .

Etymology: The species was named as "inopinata" meaning surprising or unexpected in Latin, because of its surprising biological and phylogenetic characters.

Materials examined: Holotype male, four paratype males, and five paratype females were deposited in the USDA Nematode Collection, Beltsville, MD, USA, and five paratype males and five paratype females are deposited in the Forest Pathology Laboratory Collection of Forestry and Forest Products Research Institute (FFPRI), Tsukuba, Japan.

The wild type strain NK74SC is available on request from Miyazaki University (T. Kikuchi) or FFPRI (N. Kanzaki).

Locality: The wild type strain was collected from the figs of a single tree of Ficus septica Burm.f. obtained from Ishigaki Island, Okinawa, Japan (GPS: $24^{\circ} 24^{\prime} 38.06^{\prime \prime} \mathrm{N}, 124^{\circ} 11^{\prime} 06.81^{\prime \prime} \mathrm{E}, 71 \mathrm{~m}$ a.s.l.) on 6 June, 2013.

Diagnosis: New species is characterised by the presence of short stomatal flaps, slender body, short female tail, and rounded appendage on anterior cloacal lip and tongue-like postcloacal appendage of males (Fig. 1, Supplementary Fig. 1). The combination of these characters has not been reported in the other species in the genus. Molecularly, the new species is readily distinguished from its closest relative, C. elegans by its molecular barcode sequences, and clear phylogenetic separation (Supplementary Fig. 2a and 2b).

Description. Adult: Typological characters are and the morphometric values are provided in Fig. 1, supplementary Fig. 1 and supplementary Table 1. Large and slender species; ca. 1.5-2.5 mm in length, and individuals may reach up $3.0 \mathrm{~mm}$ under optimal culturing conditions. Cuticle is moderate to thick with four-lined lateral field. Deirids on the lateral field, at the level slightly behind the secretory-excretory pore. Lip separated into six sectors, not clearly offset. Six labial sensilla and four cephalic sensilla present. The anterior end of each lip sector very slightly elongated and forming six stomatal flaps. Amphid small, oval pore-like, at the level of the margin of cheilo and gymnostom. Tube-like stoma separated into three parts; short tube-like cheilostom; simple tube-like gymnostom, which is weakly separated into two subsections; and tube-like stegostom covered by pharyngeal sleeve, which is separated into four subsections, prostegostom, mesostegostom, metastegostom, and telostegostom. Metastegostomatal three teeth flap-like. Pharynx separated into four sections; procorpus forming muscular tube, well-developed metacorpus (median bulb); glandular and narrow isthmus; and basal bulb with double haustrulum as the glottoid apparatus. Pharyngointestinal valve (cardia) prominent. Nerve ring around the middle of isthmus. Excretory pore located around the margin of isthmus and basal bulb.

Female: Gonadal system didelphic, amphidelphic. Anterior and posterior gonadal system are basically symmetric with each other, arranged as ovary, oviduct, spermatheca, spermathecal-uterus junction tissue, uterus and vulva/vagina from distal. Sometimes more than 20 developing eggs are deposited. Tail conical or forming slightly elongated conus with pointed tip. Anus and rectum clearly visible; three (two subventral and one dorsal) rectal glands present. Phasmid forming small pore at ca. $60 \%$ of total tail length from anus.

Male: Testis single, anteriorly reflexed rightwardly. Vas deferens occupying ca. 1/5 of total gonadal length. Tail enveloped by a closed bursa, supported by nine pairs of bursal rays. Anterior cloacal lip with a rounded and sclerotized appendage and bulgelike appendage between rounded appendage and cloacal opening; a small sensilla-like papilla on the bulge-like appendage. Posterior cloacal lip with tongue-like appendage with two cloacal sensilla. Spicules paired, separate, long and slender with evenly slightly ventrally curved blade and simply pointed tip. Gubernaculum slender, ventrally arcuate with small squared appendage at the distal end in lateral view. Bursa heart-shaped in ventral view, anteriorly closed with serrated edge; serratae obvious in anterior half and vague in posterior half; terminal notch present but unclear. The nine pairs of genital papillae or bursal rays supporting the bursal velum with an arranged $(2 / 1+1+2+3)$.

Morphological and ecological characteristics. C. inopinata has typological characters in common with the Elegans group of the Caenorhabditis genus, e.g., the heart-shaped male bursa which is anteriorly closed and with serrated edge ${ }^{3,4}$. Phylogenetic analyses of C. inopinata with 26 other Caenorhabditis species using small subunit (SSU) and large subunit (LSU) ribosomal RNA genes or 11 conserved genes (genes coding SSU and LSU rRNA, orthologues of C. elegans ama-1, lin-44, par-6, pkc-3, ZK686.3, W02B12.9, ZK795.3, Y97E10AL.2, and Y45G12B.2a) ${ }^{3}$, both support that $C$. inopinata is the most closely related to C. elegans among known species in the Caenorhabditis genus (Supplementary Fig. 2a, b). Regardless of its phylogenetic closeness to $C$. elegans, C. inopinata has substantial morphological differences from the other Elegans group species (Supplementary note 1). For example, $C$. inopinata has a long and slender body, reaching up to nearly $3 \mathrm{~mm}$ in length, more than twice the body length of $C$. elegans adults (Fig. 1a, Supplementary Table 1). Also, C. inopinata has a modification of the lip region (presence of very short flap-like extension of lip sector) and the female has a shortened, 
a

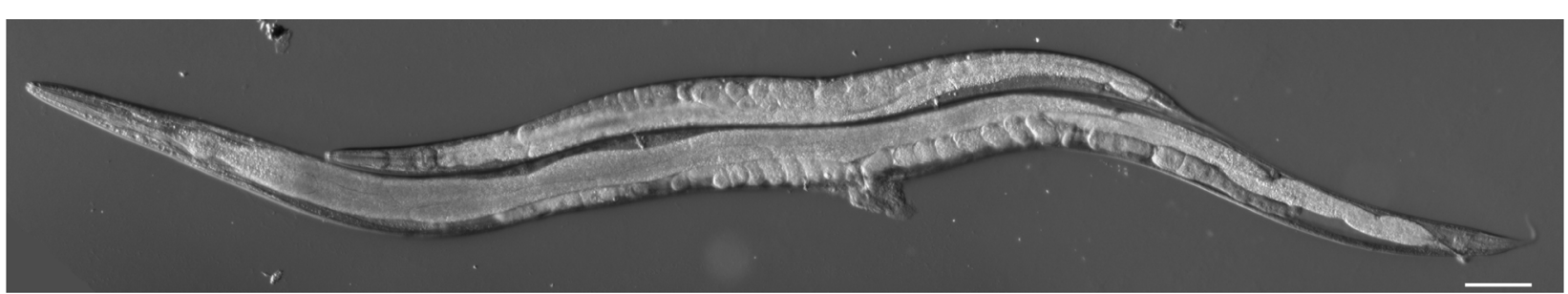

b

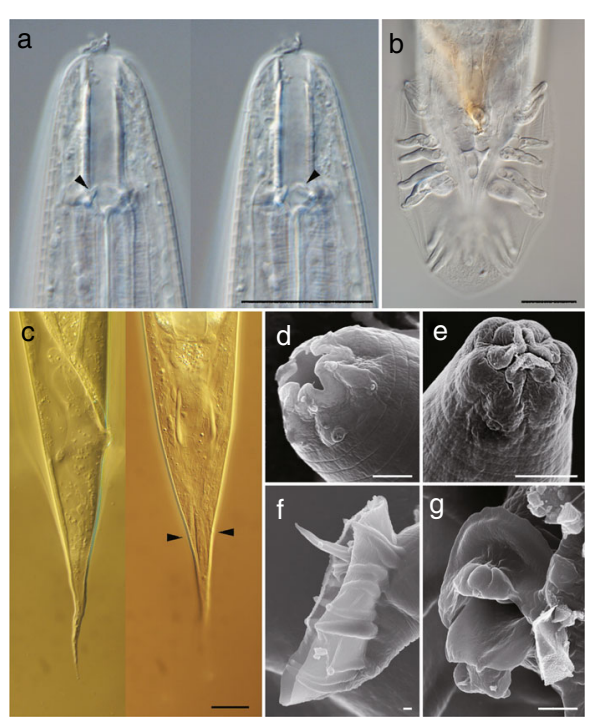

C

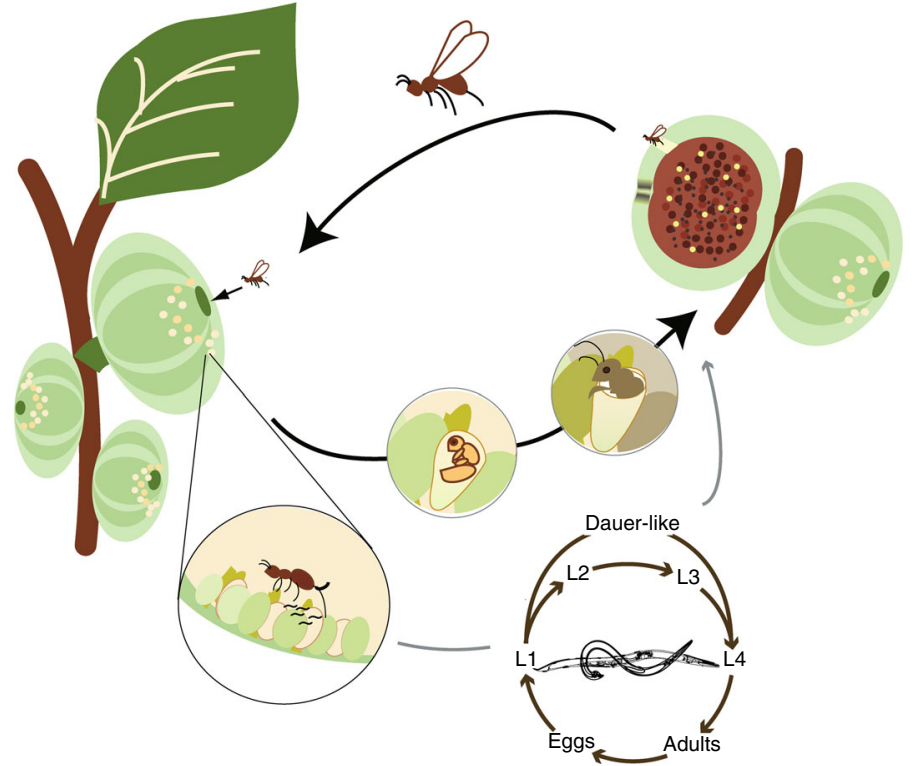

Fig. 1 C. inopinata biology compared with the sibling species C. elegans. a DIC microscopic views of C. inopinata (adult female; bottom) with C. elegans (hermaphrodite; top). Scale bar: $100 \mu \mathrm{m}$. b Key morphological characters of $C$. inopinata. a: right lateral view of stomatal part in different focal planes showing dorsal tooth (left) and right subventral tooth (right) with arrow heads. b: ventral view of male tail. c: female tail in right lateral (left) and ventral (right) view showing phasmids in arrowheads. d: face view of stomatal part of adult. e: face view of stomatal part of dauer-like juvenile. f: left lateral view of male tail. g: ventral view of male cloacal opening. Scale bars: $20 \mu \mathrm{m}$ for a-c, $2 \mu \mathrm{m}$ for $\mathrm{d}-\mathrm{g}$. c Life cycle of $C$. inopinata. The nematodes multiply in fresh syconia of Ficus septica, which has a mutualistic association with the pollinating wasp Ceratosolen sp. When the syconia mature nematodes in the dauer form disperse to new young syconia using the wasps as a vector

conical tail compared to C. elegans (Fig. 1b). These morphological differences, particularly the relatively large body, have been found in other fig-associated nematodes (Supplementary note 1) and may be adaptations to $C$. inopinata's habitat environment.

Figs and fig wasps have a mutualistic relationship essential to their survival ${ }^{5}$. C. inopinata is likely to have a close association with the fig F. septica and its pollinating Ceratosolen wasps, because the nematode was only observed either inside the fig syconium or as dauer stage larvae around the abdomen area of the adult pollinating wasps, after emerged from the syconia (Supplementary Table 7). Also, a higher frequency of C. inopinata was found in figs containing wasps than figs without wasps (Supplementary Table 6). Despite our extensive sampling in Okinawa islands, no C. inopinata or Caenorhabditis worms were detected in other Ficus species (e.g., F. variegata, F. benguetensis and F. superba) sharing the habitat with $F$. septica. These observations suggest a natural history where $C$. inopinata proliferates in F. septica syconia and disperses from matured to new syconia using the fig wasps (Fig. 1c).

Like C. elegans, C. inopinata is well suited to rearing under laboratory conditions, and can be cultivated using the standard C. elegans culture method (NGM + E. coli OP50). When $C$. inopinata were raised on E. coli HT115, gonadal elongation was enhanced (Supplementary Table 3) and brood size of $C$. inopinata became larger than those on OP50. OP50 and
HT115 are known to have different nutritional qualities, and affect longevity and development of C. elegans of certain genetic backgrounds $s^{6-8}$. Because the effect of these bacteria on $C$. inopinata were different from that of C. elegans, it is likely that the two nematode species have distinct nutritional responsiveness. C. inopinata has mouthparts characteristic of bacterivorous nematodes (Fig. 1b, Supplementary note 1) and the intestine of freshly collected wild C. inopinata adults contained a wide variety of bacterial species (Supplementary Table 4). Nematode population growth was similar or elevated when $C$. inopinata were raised on the bacteria isolated and cultured from the C. inopinata intestine, compared to growth on E. coli (Supplementary Fig. 3a). Together, these results suggest the main nutrient source of the nematodes is bacteria associated with the fig syconia. Metabolic pathway analysis and CAzyme analyses based on genomic data (see Genome structure and contents section below, and Supplementary note 3) revealed a similar pathway structure and genes involved in energy metabolism for $C$. inopinata and C. elegans, also supporting C. inopinata bacterivory.

The optimal culture temperature for C. inopinata is $25-29^{\circ} \mathrm{C}$, significantly higher than that of C. elegans $\left(20^{\circ} \mathrm{C}\right.$ ) (Supplementary Fig. 3b), possibly reflecting the subtropical habitats of C. inopinata versus Bristol, England the source of the standard N2 C. elegans laboratory strain. 


\section{Table 1 Genome statistics}

\begin{tabular}{llll} 
& C. inopinata & C. elegans & C. briggsae \\
\hline Assembly size (Mb) & 123.0 & 100.3 & 108.4 \\
Number of scaffolds & $6+1$ & $6+1$ & 367 \\
Average (kb) & 17,573 & 14,327 & 295 \\
Largest scaff (kb) & 23,638 & 20,924 & 21,541 \\
N50 (kb) & 20,595 & 17,494 & 17,485 \\
L5O (n) & 3 & 3 & 3 \\
Gaps (bp) & 413,509 & 0 & $2,967,626$ \\
GC (\%) & 38.47 & 35.44 & 37.35 \\
Num. coding genes & 21,608 & 20,247 & 21,814 \\
Coding gene size (median; bp) & 1992 & 1972 & 1964 \\
Total coding genes (Mb) & 66.0 & 63.3 & 64.7 \\
Protein similarity & $81.3 \%$ & $\mathrm{NA}$ & $80.4 \%$ \\
(vs. C. elegans) & & & \\
Synteny coverage & $76.3 \%$ & $\mathrm{NA}$ & $68.7 \%$ \\
(vs. C. elegans) & & & \\
Complete BUSCOs (protein) & $98.1 \%$ & $99.6 \%$ & $98.4 \%$ \\
\hline
\end{tabular}

Genome structure and content. Like C. elegans and other Caenorhabditis species ${ }^{9}, C$. inopinata has six pairs of nuclear chromosomes $(2 n=12)$ (Supplementary Fig. $4 \mathrm{a})$. The C. inopinata genome assembly was produced by combining multiple sequencing technologies and manually finished into six chromosomes and one mitochondrial genome (Table 1) (Supplementary note 3 ). This 123 megabase (Mb) assembly is the second nematode species after $C$. elegans ${ }^{10}$ whose genome sequence information comprise solely chromosomes. By resequencing the males and females of C. inopinata (Supplementary Fig. 4b), we have shown that $C$. inopinata possesses an XO sexual system. Mating experiments show a slightly female-biased progeny sex ratio (Supplementary note 2 ). Female-biased sex ratios have also been observed in C. briggsae crosses $^{11}$, while 1:1 sex ratios are observed in $C$. elegans crosses. Female-biased sex ratios are selected for under local mate competition, which $C$. inopinata is likely to be experiencing because colonisation in a fig is mostly by relatives (Supplementary note 2 and ref. ${ }^{12}$ ). Alternatively, it could be caused by other mechanisms, including sperm competition $^{11}$.

A total of 21,609 gene models were predicted in C. inopinata aided by transcriptome sequencing of various life stages and extensive expert curation (Table 1). Of these, $>95 \%$ gene models were orthologous to C. elegans and C. briggsae genes involved in well-studied biological pathways, including insulin/insulin-like growth factor signalling, sex determination, dauer formation, small RNA pathways, and nuclear receptors (Supplementary Table 15-19), indicating essential genes among the three species (C. elegans, C. briggsae and C. inopinata) are well conserved, and supporting a high quality of our gene predictions in the $C$. inopinata genome. Orthologue clustering was inferred between $C$. inopinata and nine other Caenorhabditis species, confirming its closest relationship to $C$. elegans (Fig. 2a). Divergence time estimation placed the separation of the two species 142.73 million generations ago. Assuming average generation time of 30 days, $C$. inopinata diverged from C. elegans about 10.5 mya (Supplementary Fig. 4c, Supplementary note 3 ).

Although the vast majority of $C$. elegans genes were conserved, a small number of important $C$. elegans genes were missing in $C$. inopinata. For example, among the sex determination genes, the orthologue of C. elegans her-1 was apparently disrupted by LTR insertion (Supplementary Table 17). Additionally, although $C$. inopinata has FTR ( $f \circ g$ 2-related) gene family to which fog-2 belongs, the orthologue of fog-2, an essential gene for hermaphroditism in C. elegans, was absent in C. inopinata (Supplementary Table 17), as observed in C. briggsae ${ }^{13}$.
Experimental tractability. The high level of genetic similarity between $C$. inopinata and $C$. elegans allows many molecular techniques and resources used for C. elegans to be directly applied to $C$. inopinata studies. Stable transgenic lines of C. inopinata were successfully obtained by germline microinjection of marker plasmids commonly used in C. elegans, including rol-6(d) (dominant Roller phenotype), SUR-5::NLS::GFP (universal nuclear marker), Podr-1::GFP (AWC neuron marker), Punc-122:: GFP/RFP (coelomocyte marker), and Pmyo-3::mCherry (body wall muscle marker). All $C$. inopinata transgenic lines showed similar phenotypes (Rol) or expression patterns to those found for C. elegans (Fig. 3a), suggesting that transcriptional regulation by promoters and post-transcriptional regulation by untranslated regions (UTRs) are highly conserved and compatible (Fig. 3b). In addition, a panel of antibodies against $C$. elegans proteins successfully recognised analogous structures in $C$. inopinata by immunofluorescence. For example, antibodies against $C$. elegans TBG-1 $(\gamma \text {-tubulin })^{14}$ recognised centrosomes in early $C$. inopinata embryos (Fig. 3c) as in C. elegans embryos, suggesting that the antibodies recognised the $C$. inopinata TBG-1 ortholog (amino acid identity to C. elegans TBG-1: 97.5\%). Antibodies against C. elegans PGL-3 ${ }^{15}$, a core component of $\mathrm{P}$ granules (germ granules) and one of the rapidly evolving proteins, also recognised $\mathrm{P}$ granule-like structures in C. inopinata embryos (amino acid identity between $C$. elegans PGL-3 and its $C$. inopinata ortholog: 46.8\%) (Fig. 3c). Considering the high conservation of transcriptional and post-transcriptional regulatory sequences and amino acid sequences between $C$. elegans and C. inopinata, rich resources developed for $C$. elegans, including vector plasmids used for transgenesis and antibodies are likely to be directly usable in C. inopinata, which makes this organism a suitable comparative model to $C$. elegans at genetic and molecular levels.

Chemoreceptors in C. inopinata. Gene family and protein domain analyses (see Supplementary note 3) suggested that there are considerable contractions in seven-transmembrane $\mathrm{G}$ proteincoupled receptor (7TM-GPCR) (also called serpentine) gene families in $C$. inopinata. By intensive gene curation (Supplementary note 3 ), we identified a complete gene list of $C$. inopinata serpentine receptors comprising 382 genes and 48 pseudo or partial genes (Fig. 4a, Supplementary Table 20), a number significantly smaller than that of $C$. elegans (1329 genes) $(p<2.2 \mathrm{e}$ -16 , Chi-squared test) and slightly smaller than C. briggsae (452 genes) (Fig. 4a). It has been reported that the gene number differences between $C$. elegans and other Caenorhabditis species were the result of gene expansion in $C$. elegans rather than gene losses in the other species ${ }^{16}$. Our phylogenetic analyses, however, suggested that massive gene losses clearly occurred in C. inopinata, such as srd, sre, srh, and srt serpentine families (Fig. 4b, Supplementary Fig. 6). These analyses also indicate that local gene expansions have occurred in specific subgroups in C. inopinata serpentine families, overall, retaining the total serpentine gene number to $\sim 400$ (Fig. 4b, Supplementary Fig. 6). These results suggest that $C$. inopinata requires a narrower range of receptors, compared to the 'soil/rotting fruits' nematodes (C. elegans and $C$. briggsae). A contraction of GPCR families could be related to its limited habitat area and close association with the fig and vector insect, in which fine detection of only a small variation of chemical stimuli may be required (Fig. 1b).

To probe the chemosensory response of $C$. inopinata to ethologically relevant molecules, we examined ascaroside signalling. C. elegans and other nematodes produce a wide range of ascarosides (secondary metabolites that are glycosides of the dideoxy sugar ascarylose); many behaviours are affected by particular combinations of ascarosides and many GPCRs are 
a

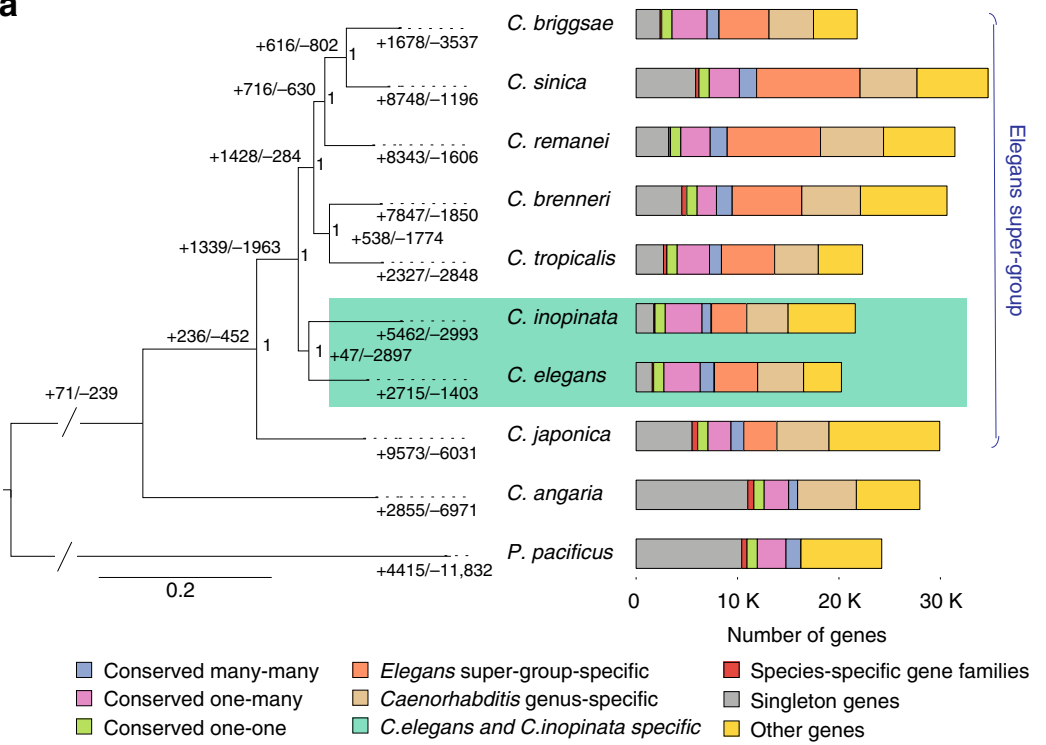

b

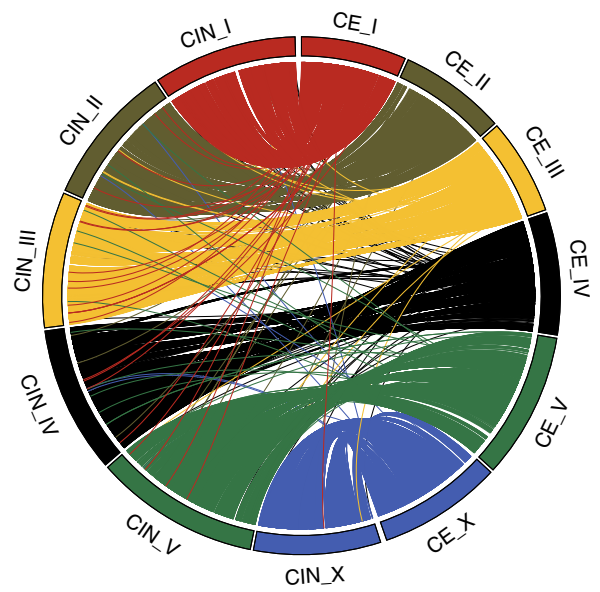

Fig. 2 Genome of C. inopinata compared with C. elegans and other relatives. a Phylogeny and gene family evolution of Caenorhabditis species. Numbers labelled on each branch (or lineage) are the specific gain/loss of that branch (or lineage). Genes are categorised in stack bar, and the length of stack bar is proportional to number of genes. b Single copy orthologues linking C. inopinata and C. elegans chromosomes

implicated in the ascaroside response ${ }^{17}$. We found that mixed stage $C$. inopinata cultures produce many of the key ascarosides found in C. elegans (Supplementary Fig. 3c) and C. inopinata males respond to the prominent ascarosides (Fig. 4c). C. inopinata males are more sensitive to the tested ascarosides than are C. elegans males, for example, responding to a lower amount of the ascr\#3 (Fig. 4c). We conclude that ascaroside-mediated mate recognition is conserved between these sibling species but there are evolutionary differences of the details of that signalling, potentially reflected in their GPCR repertoire.

Expansion of transposable elements in $C$. inopinata. The $C$. inopinata nuclear genome is $23 \mathrm{Mb}$ larger than its sibling $C$. elegans (Table 1, Supplementary Table 9) possibly attributable to genome shrinkage associated with the evolution of selfing. Previous comparative genome studies of selfing and male/female species suggested genome degradation occurs in selfing species because of adaptive gene loss and relaxed selection on genes for the maintenance of sexual traits ${ }^{18,19}$. Recently, direct comparison of $C$. briggsae and her sister male/female species C. nigoni, confirmed extensive gene loss (up to $16 \%$ ) in C. briggsae ${ }^{20}$. While gene loss may have contributed to the genome size differences, the magnitude is likely low because $\sim 95 \%$ of $C$. inopinata genes have C. elegans orthologues. Another possibility is skew, a nonadaptive process of biased chromosome transmission, whereby fathers transmit the shorter allele of an autosomal chromosome pair preferentially to their daughters ${ }^{21}$. Skew is predicted to passively drive genome reduction in selfing nematodes and likely occurs in all Caenorhabditis species ${ }^{22}$. Consistent with this model is that intergenic distances on C. elegans autosomes are shorter than on their X chromosomes and shorter than on C. inopinata autosomes. While genome reduction has likely occurred in $C$. elegans, a third explanation is C. inopinata genome expansion. Indeed, the proliferation of transposons and other repetitive elements, in particular LTR retrotransposons, long interspersed nuclear elements (LINES) and Tc1/mariner transposons, in the intergenic regions are highly expanded in the $C$. inopinata genome compared to C. elegans and C. briggsae and may be the main driver for the genome size differences between these species (Supplementary Table 10, Extended Figure 7b). The C. inopinata genome contains 641 LTR retrotransposon elements, 104 of which contain full protein domains (intact LTRs) In comparison, C. elegans and C. briggsae have 62 and 128 LTR retrotransposon elements, respectively, with 10 intact LTRs found in each (Fig. 5a). Phylogenetic analyses of reverse transcriptase (RT) domains of LTR retrotransposon elements from C. inopinata, $C$. elegans, and $C$. briggsae, showed that three families of LTR elements have expanded in C. inopinata (Ty3/Gypsy, Bel/Pao LTR families and RVT_1) (Fig. 5b). All three phylogenetic clades contain between 3 and 20 sequences from both $C$. elegans and $C$. briggsae showing close relationships to C. inopinata RTs (Fig. 5b). This suggests that the expansion of LTR elements in C. inopinata is unlikely a result of new acquisition of LTR elements from an external source but mainly due to replication of elements that existed in a common ancestor.

The most common type of repetitive sequence in the $C$. inopinata genome is the TcMar transposase Tc1 family which has expanded in C. inopinata, comprising $8.85 \%$ of the genome, compared with $1.31 \%$ and $3.04 \%$ of the C. elegans and C. briggsae genomes, respectively (Supplementary Table 10). Phylogenetic analyses show that the $C$. inopinata TcMar transposases form five main clusters, which are related to $C$. elegans Tc1 and Tc3 transposons, C. briggsae Tcb1 and Tcb2 transposons, Mos1 transposons from Drosophila and HsMar transposons from humans (Supplementary Fig. 5). Together, these results suggest that there is a high level of transposable element activity in the $C$. inopinata genome, compared with other Caenorhabditis species, and this could be a driving force of the evolution of $C$. inopinata.

Orthologues of three C. elegans genes (ergo-1, eri-6/7, and eri-9) involved in the ERGO-1 26G small-interfering(si)RNA pathway are missing in $C$. inopinata, although other siRNA/microRNA related pathways are highly conserved in the three Caenorhabditis species (Fig. 5c, Supplementary Table 19). The absence of those genes were confirmed from several aspects of evidence including BLAST search against the gene models, genome, and transcriptome data. In C. elegans, the ergo-1 pathway is likely involved in targeting and suppressing the expression of newly acquired, duplicated genes, possibly of viral origin ${ }^{23}$ and potentially deleterious non-coding regions of the genome ${ }^{24}$. Gene synteny in the ergo-1 region of the genome is highly conserved between $C$. 
a

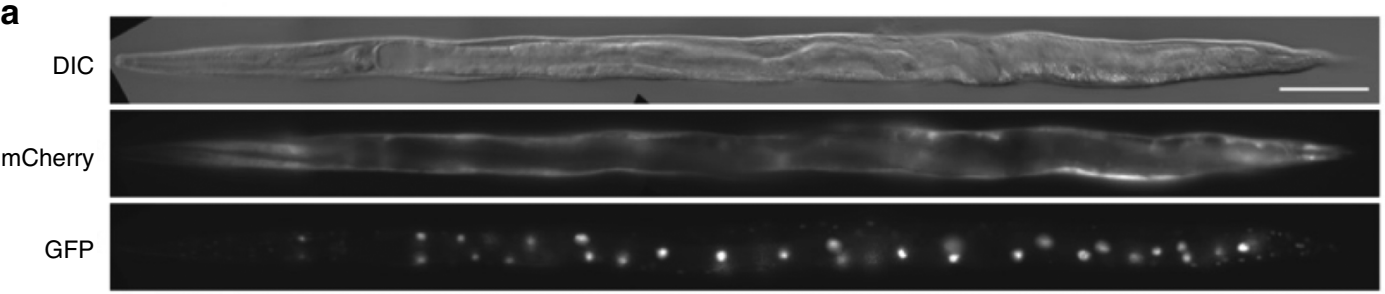

b

Cel-sur-5 GCA---TAGAACAGTGATCAATGTGTTGTGCTCACTAGTCAATGATGTTCGTCTCTCATTTTTTCCTACAACTTCACTGAAAAGGTTTTGAATATCTGGTGATTGTAGGCAATCACACCAC Cin-sur-5 CAAAATTAGAACAGCGATAAATGTGTTTCGCTCTGCAATCAGTGATGTTCGCATGCAGTCTG----GAAAATTCAACGAAAAATGCTTGAATATCTGGTGATTGTTGGCAATCACACCAT

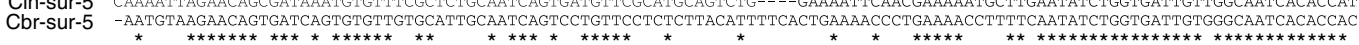

AGTTAATGGCCTGGCAAATA-ATGATAAGCGTGTGATAAACCTCACAAAAATACGCCT----AGAAGTGGTGATATCG-CTAGTTTTTGTGTCCTTTCGATTTTTTTTCGTGTTGCTITTTTT AGTTAATGGCCTAGCAAATA-AAGATAAGCATTTGATAAACCTGCCAAAAAATGGCCCTAAAAGAAGTGATGAAATAG-CAGAGTTTCCTTGTTIATCTGGCGTTTTTTGTTAAACCTATT

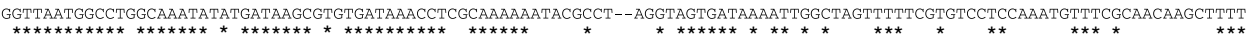

CTGTGGATAAACCTTTGAACTTTACATTTTGTTTTCAGAATG TT-TAGATAAATGTTTGAACTTTAAAA---AATTTCAGAATG

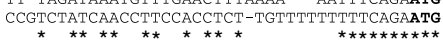

Cel-myo-3 GAGAGAGAGTGCAGAGAGGGCGGGATAGCTCCCGGGATTCCGTTTTCTTCTTCTTTATCTTCAACGATGATGTGTGTGCGTGT---------TGTATAGATTCTGTTGCTCCCCC-ACAAC Cin-myo-3 TGAGGAGAGTGCAGAGAGGGCGGGT--GCTCCCGGGC---CTTCTGCTTCTTATTCATCTCCAGCGATGATGTGTGTGCGTGTGTATGTGCTATATACATTCTGTTGCTCCCCCCACAT

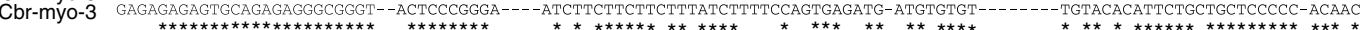

TC-GCTCCGAAGGCTCAATACAATTCAATTGATATTGGA--------GGAGAGCCTACCGG---AGTGGGAGGATAAGAAGAAACATAAGAAGAAGAAGAAGAAGAAG-- TC-GCCCCG- - -GCTCAATACAATTCAATTGATATTAGGAGGGGAGGGGAGAAGCTACCGGGGCAGTGGGAGGAGAAGAAGAAACATATGAAGAAAAAGAAGGTGAAGGTGATGAAGAAG

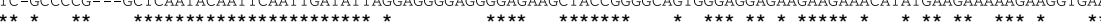
-----CATGCTTCTGGTTTTTTGATGCTATGAAAACGGCA-----CAAAAAGATGATTGAGGTC-----CCTTTTTCAATACC-TTCTCTCATCT-TTCAAATCCCATTGAAACCTAAAAC

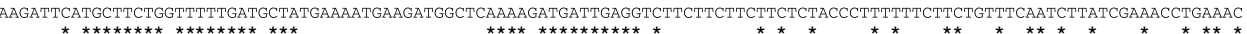

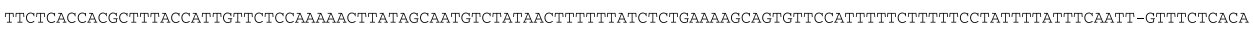
ATTGCCCCTTTTCTAACTATTCATTTCCC---GACCATCGC-TTCCATTTAAATTTATAGGCAACTA---CGTCTTTCTTTATTTTTTTGTTAAGATTTTTCCATGACCCCTTTATC--A

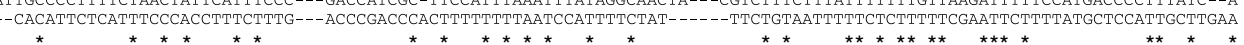

TTTCGTTTGGATTCTTTGCTTGTCAACCAGCT-TCTTCTTCCACTTTTACCGTCTAATTTTCAGGGCAGGGAGCCATCAAACCCACGACCACTAGATCCATCTAGAAATG TTGCAATTTTACCTCITCCACAATACTCATTCGTATATTGAGCAATGTGACATTTGAATTATTTGTTGTAGGGTCGGCAG--CCACGACCACTAGATACATCTAGAGATG GTGAAACATAATCACTCGCTTCTAACTTCAAT-TATTCTAAGAACTTT-----TTAATTACAGGGCAGGGAGCCATCAAACCCACGAACACTAGGTCCATCTAGAAATG

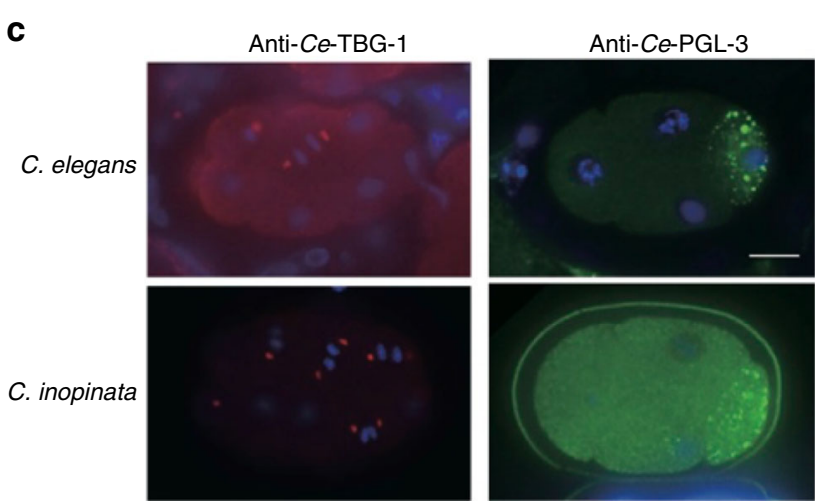

Fig. 3 Conservation of regulatory sequences and amino acid sequences between C. elegans and C. inopinata. a Transgenesis in C. inopinata using C. elegansderived transgene. Ce-MYO-3::mCherry (body wall muscle: bottom) and Ce-SUR-5::GFP (somatic nuclei: bottom) signals in an adult female animal are shown. Scale bar: $100 \mu \mathrm{m}$. b Comparison of promoter regions of the sur-5 and myo-3 gene. c Immunofluorescence using antibodies against $C$. elegans proteins. Anti-Ce-TBG-1 (red) stains centrosomes, and anti-Ce-PGL-3 (green) stains P granules (germ granules) in both C. elegans and C. inopinata embryos. Blue: DAPI. Scale bar: $10 \mu \mathrm{m}$

elegans and C. briggsae. In C. inopinata, the gene order in the analogous region of the genome has undergone some rearrangements, including the loss of ergo-1 and hypothetical gene K10C9.1/ $\operatorname{cbg} 17878$, as well as expansion of several gene families, including mariner-like transposase (TcMar) and RT genes (Fig. 5c), which was confirmed by a long-range PCR targeting this region in $C$. inopinata using primers (ci_R09A1_f: CACATTTCGGTACTTGCCAAT and ci_Y50D4C_r: GGAATT TGATGGCATGGTT) and subsequent Sanger sequencing. Gene rearrangement in the ergo-1 region could therefore be a consequence of DNA transposase and LTR retrotransposase activity. If the ergo-1 pathway has a role in suppressing transposases, the loss of ergo-1, could have further enhanced transposase activity through the reduction of $26 \mathrm{G}$ siRNAs in the C. inopinata genome.

We found that $C$. inopinata, like the $C$. elegans $\mathrm{N} 2$ strain, was highly susceptible to RNA interference (RNAi) by ingestion of double-stranded RNA in both germline and soma, unlike other Caenorhabditis species including C. briggsae, C. remanei, and C. brenneri, and some $C$. elegans natural variants ${ }^{25}$. Feeding RNAi for C. inopinata unc-22 orthologue resulted in $93.9 \%$ of F1 worms showing a twitching phenotype, similar to C. elegans unc-22 mutants. Feeding RNAi against GFP transgene to a GFP $C$. inopinata transgenic line reduced GFP signal by $\sim 80 \%$ (Fig. 6a, b), although neuronal cells appeared less sensitive. Soaking and feeding RNAi for $t b g-1$ ( $\gamma$-tubulin) orthologue of $C$. 
a

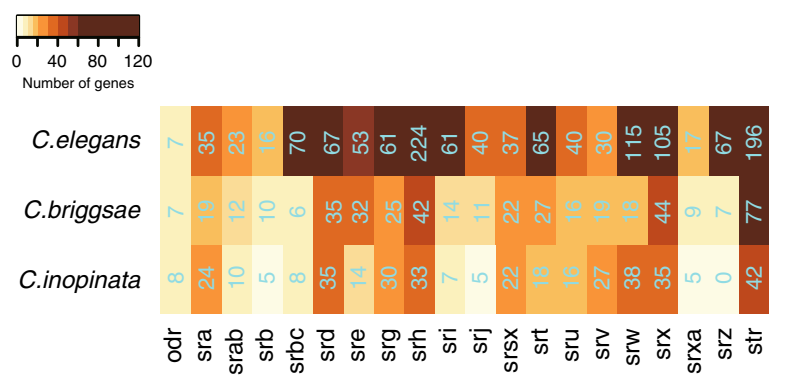

C

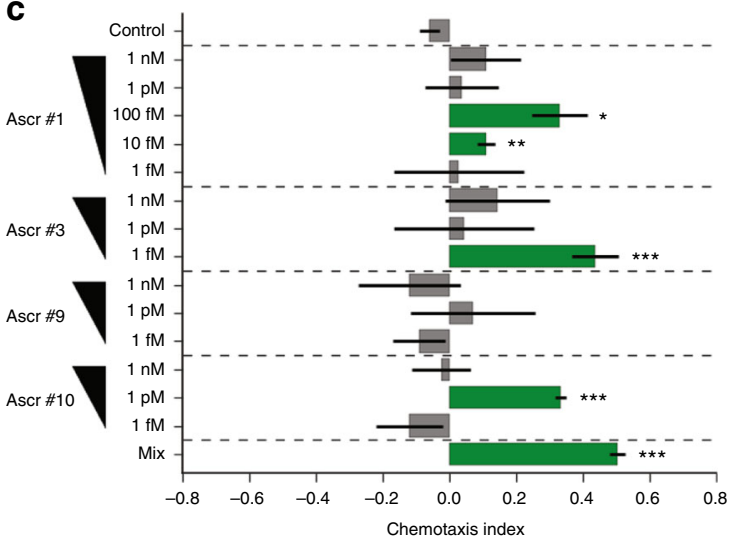

b
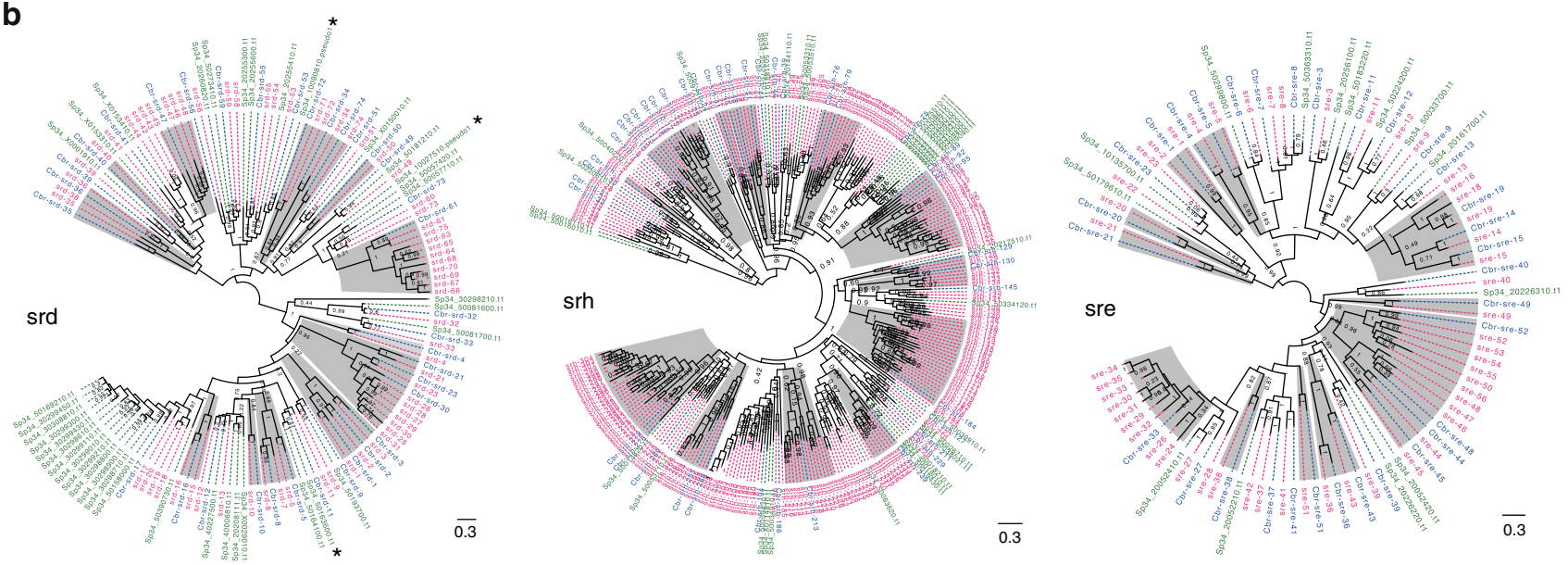

C. inopinata C. elegans C. briggsae

Fig. 4 Diversity of 7TM GPCR gene families in C. inopinata and chemosensory response. a A heat map showing gene numbers of 7TM GPCR (serpentine) gene families in C. inopinata, C. elegans, and C. briggsae. b Maximum likelihood trees of srd, sre, and srh serpentine families of the three Caenorhabditis species showing gene losses, as well as local gene expansions in C. inopinata. Clusters with gene loss in the C. inopinata genome were shaded in grey. c Response of C. inopinata males to synthetic ascarosides produced by mixed stage cultures. $\mathrm{Cl}$ chemotaxis index. Ascr\#, refer to distinct synthetic ascarosides that are made by $\mathrm{C}$. inopinata. The bars represent the mean $\mathrm{Cl}$ and the whiskers are the SEM. The green-coloured bars indicate ascaroside concentrations that were significantly different from the control

inopinata resulted in arrested early embryos with cell division defects (Fig. 6c), which is similar to the C. elegans tbg-1(RNAi) phenotype. Together, these results demonstrate efficient levels of gene silencing by RNAi methods, implying that the dsRNA uptake pathway, including SID-2, is conserved in C. inopinata and C. elegans, unlike other Caenorhabditis species including $C$. briggsae, C. remanei, and C. brenneri, which have diverged SID-2 functions $^{25}$. In C. elegans, exogenous-RNAi and endogenousRNAi use a common downstream pathway for target silencing. In C. elegans, loss of function of genes involved in the ERGO-1 class 26G RNAs pathway causes an Eri (Enhanced RNAi) phenotype, which shows enhanced sensitivity to exogenous RNAi reviewed in ref. ${ }^{26}$. Therefore, the loss of key genes involved in ERGO-1 class 26G RNA pathway (ergo-1, eri-6, and eri-9/10) may also account for the high exogenous RNAi efficiency observed in C. inopinata.

Genome structure evolution. The completeness of the C. inopinata reference genome allowed us to investigate the chromosome evolution in Caenorhabditis, especially revisiting the arm/ centre definition ${ }^{27,28}$. Seventy-six percent of the C. inopinata genome can be assigned to blocks of collinear genes (synteny) based on the position of the orthologues between the three completed Caenorhabditis genomes (Table 1). Gene collinearity is largely conserved despite frequent intra chromosomal rearrangements (Fig. 2b, Supplementary Fig. 7a). The positions of rearrangements on autosomes appear to be independent of species relatedness, as well as chromosome arm/centre definition (Supplementary Fig. 7a). Consistent with the gene family analysis, regions without synteny (breaks) in the $C$. inopinata genome are likely due to expansion of gene families enriched in transposonrelated GO terms (Supplementary Table 21). Conversely, synteny breaks in C. elegans are mainly due to an expansion of GPCRs gene family especially on chromosome V (Supplementary Table 21).

We observed significantly higher synonymous substitution rates $(\mathrm{dS})$ in autosomal arm regions than in the centre regions for C. elegans and C. inopinata one-to-one orthologues. No difference was found between the arm and centre regions of chromosome $\mathrm{X}$, and overall $\mathrm{dS}$ was lower in chromosome $\mathrm{X}$ than autosomes (Supplementary Fig. 7e). These observations suggest that intra-chromosomal translocation of Caenorhabditis genes may have little fitness cost, but the evolutionary processes acting upon them, as determined by $\mathrm{dS}$, is dependent on the region of the chromosome and whether the chromosome is an autosome 

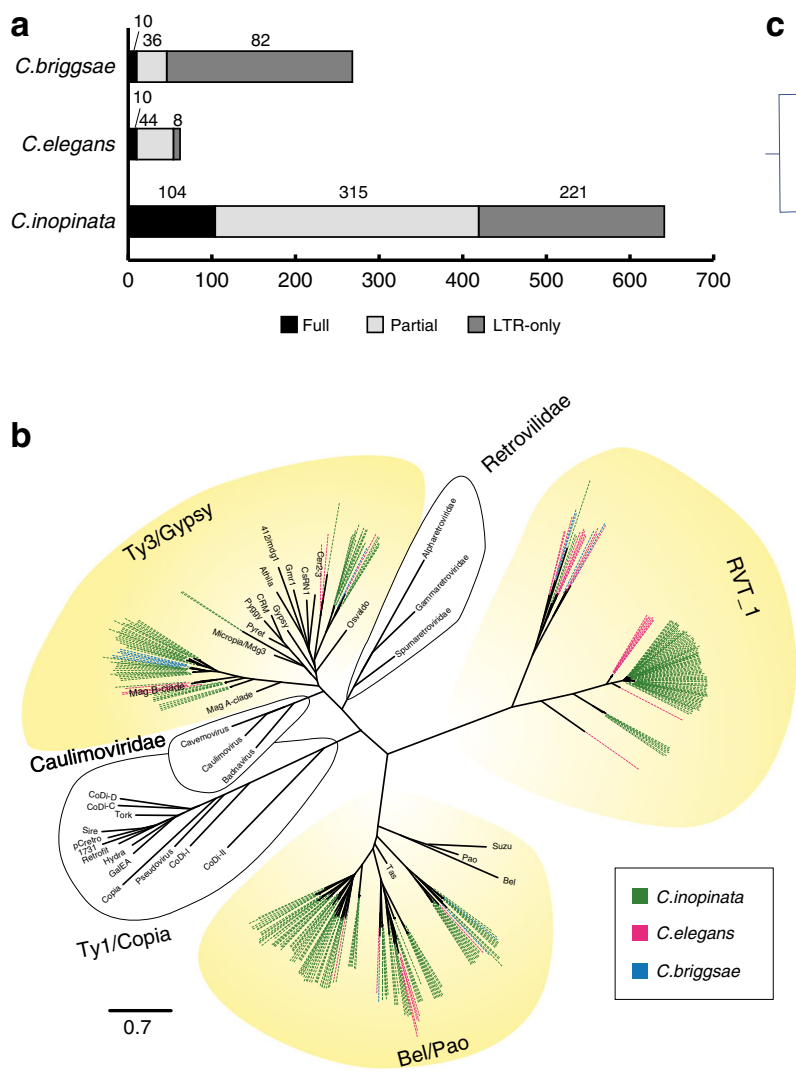

C

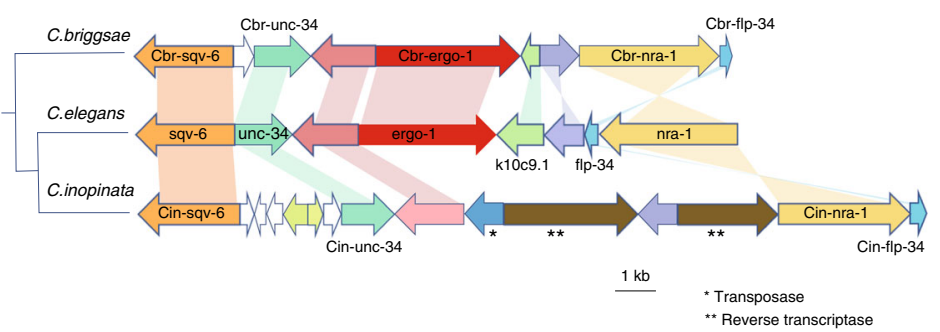

Fig. 5 Transposon expansion and ergo-1 gene loss in C. inopinata. a Number of LTR retro-transposable elements in C. inopinata, C. elegans, and C. briggsae. LTR elements were grouped into 'Full' with full LTR retrotransposon domain sets (reverse transcriptase; RT, protease, integrase, RNase H), 'Partial' with at least one of the domains but not full, and 'LTR-only' having only LTR regions with no protein domains. $\mathbf{b}$ Phylogenetic relationships of LTR retrotransposons in the three Caenorhabditis species. RT domains extracted from LTR retro-transposable elements of the three species were aligned by Mafft v7.22152 with reference sequences obtained from GyDB (Gypsy DataBase) ${ }^{75}$ and a maximum-likelihood tree was constructed using RAxML v7.2.8 using the best-fitting empirical model of amino acid substitution with 1000 bootstrap resampling replicates with the percentage support shown on the nodes. Green, red, and blue lines on the branches represent C. inopinata, C. elegans, and C. briggsae sequences, respectively. The scale bar shows the number of amino acid substitutions per site. c Gene synteny in C. elegans, C. briggsae, and C. inopinata for regions of the genomes corresponding to ergo-1 C. elegans gene. ergo- 1 is highlighted in red and orthologous genes are grouped by colour. Genes with no orthology to other genes in the region shown are white

or sex chromosome. This can be demonstrated by the Caenorhabditis sex chromosome, which has a reduced effective population size and recombination rate re,19,29 $_{\text {Supplementary }}$ Fig. 7d, e).

In conclusion, $C$. inopinata, the sibling species of $C$. elegans, serves as a new satellite model system that will enable deep evolutionary interpretations of biological phenomena through comparative analyses with C. elegans and other Caenorhabditis spp. Characterisation of the genome, morphology, ecology, and behaviour of $C$. inopinata has revealed robust similarities, as well as substantial differences with $C$. elegans. Notably, C. inopinata has highly conserved gene synteny and orthology of essential genes with $C$. elegans and C. briggsae, but differs substantially in morphology and ecology. We hypothesise that activation of transposable elements possibly due to de-silencing through an altered small RNA pathway could be a driving force of rapid diversification of $C$. inopinata from other Caenorhabditis species. The contiguous assembly of the $C$. inopinata genome and genetic data presented here, in addition to the transferability of genetic and molecular tools available for $C$. elegans to $C$. inopinata, provides an exciting new platform to perform comparative evolutionary studies.

\section{Methods}

Nomenclatural acts. This published work and the nomenclatural acts it contains have been registered in ZooBank, the proposed online registration system for the
International Code of Zoological Nomenclature (ICZN). The ZooBank LSIDs (Life Science Identifiers) can be resolved and the associated information viewed through any standard web browser by appending the LSID to the prefix "http://zoobank. org/". The LSIDs for this publication are: urn:lsid:zoobank.org:act:70EEB2ACC773-4AC4-B73B-E59191883567.

Discovery and observation of $\mathbf{C}$. inopinata. About 30 of Ficus septica were collected manually from Ishigaki Island, Okinawa, Japan on 6 June, 2013 (GPS: 24 $24^{\prime}$ $38.06^{\prime \prime} \mathrm{N}, 124^{\circ} 11^{\prime} 06.81^{\prime \prime} \mathrm{E}, 71 \mathrm{~m}$ a.s.l.). The figs were brought back to the laboratory, cut into half with a sterilised knife, and settled individually on $2.0 \%$ agar plates $(\varnothing=90 \mathrm{~mm})$. The plates were kept at room temperature $\left(25^{\circ} \mathrm{C}\right)$, and propagation of any nematode species were examined daily. The propagated nematodes were observed under light microscope to determine their food source (bacteria or fungi), and transferred to either NGM seeded with E. coli OP50-1 (for bacteriovorus species including C. inopinata) or PDA previously inoculated with Botrytis cinerea (for fungivorous species). The wild-type strain of $C$. inopinata was coded as NK74SC, and kept as a laboratory strain.

Male and female adults from a 2-week-old culture of the strain were handpicked from the culture plate. Live worms were observed, drawn and micrographed under light microscope (Eclipse 80i; Nikon) equipped with DIC/Nomarski devices, drawing tube, and a digital camera system (DS-Ril; Nikon). Permanent slides (type materials) for measurements and morphometrics were prepared from the same culture plate used for morphological observation, where hand-picked nematodes were heat-killed, fixed in TAF, processed into glycerine using modified Seinhorst's methods and mounted in glycerine according to Maeseneer and d'Herde methods ${ }^{30}$

Establishment of an isogenic line. Gravid females from a 1-week-old culture were hand-picked and individually transferred to a small $(\varnothing=60 \mathrm{~mm})$ NGM plate previously seeded with E. coli OP50-1, and the plates were kept at $25^{\circ} \mathrm{C}$. This 
a

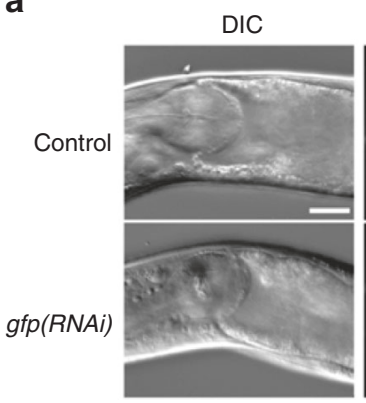

GFP

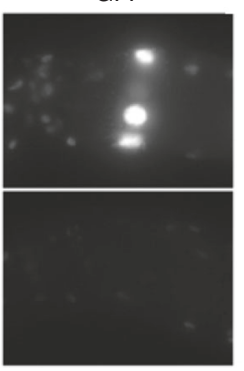

b

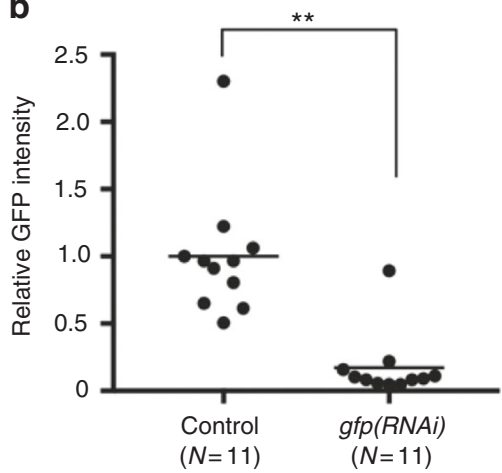

C

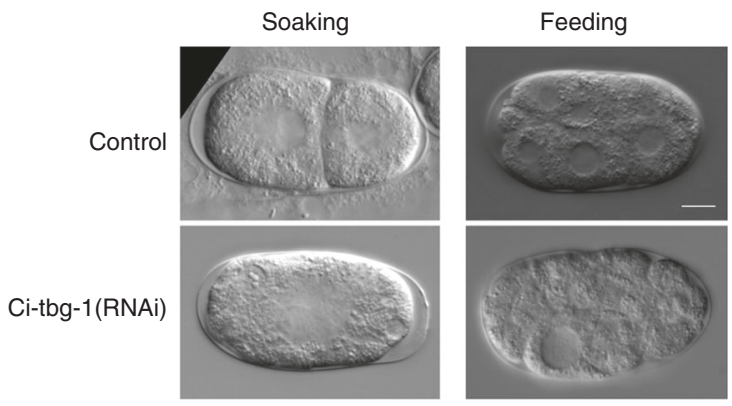

Fig. 6 RNAi efficiency in C. inopinata. a Feeding RNAi in C. inopinata. The GFP encoding transgene in a transgenic line from Fig. 3a was targeted by feeding RNAi. The GFP signal was significantly reduced. Scale bar: $20 \mu \mathrm{m}$. b Efficiency of $g f p(R N A i)$. Relative intensities of the GFP signal in control and RNAitreated worms was compared. ${ }^{\star *} p<0.01$, Student's t-test. c Soaking and feeding RNAi of Ci-tbg-1. Embryos were arrested with cell division defects (soaking RNAi: 1-cell arrest, feeding RNAi: early embryo with some abnormally large nuclei). Scale bar: $10 \mu \mathrm{m}$

procedure was repeated 10 times using a successfully propagated plate in each generation. The last generation was subcultured and kept at the laboratory as the isogenic line of $C$. inopinata with a culture code NKZ35. The isogenic line served for the successive laboratory experiments.

Genome sequencing and assembly. Illumina reads from multiple paired-end and mate-pair libraries (Supplementary Table 8), generated using DNA extracted from the C. inopinata isogenic line, were quality-trimmed using Trimmomatic v0.32 with options (ILLUMINACLIP:2:30:10 LEADING:10 TRAILING:10 SLIDINGWINDOW:4:15 MINLEN:36) ${ }^{31}$ (paired-end reads) or with the $-\mathrm{m} 50$ option $^{32}$ (mate-pair reads), and then assembled using the Platanus assembler v1.2. $4^{33}$ with options (assembly; -k 52, scaffolding and gapclosing; default parameters). Two assemblies consisting with PacBio reads (Supplementary Table 8) were produced using Falcon v.0.3.0 with options (overlap minlen 1000; to graph minlen $=8000 ;-$ max diff $\left.100-\max \_\operatorname{cov} 100-\min \_\operatorname{cov} 10\right)^{34}$ and Canu v1.3. $3^{35}$ with the default parameters. Consensus from both PacBio assemblies was improved using the Quiver module in the SMRT Analysis pipeline (version 2.3.0) ${ }^{36}$ with the default parameters. The two PacBio assemblies and the Illumina assembly were merged using Metassembler v $3^{37}$ with the default parameters. The merged assembly was further improved using HaploMerger2 v20161205 ${ }^{38}$ (default parameters on the repeat-masked assembly by RepeatMasker, see below) and SSPACE v3. $0^{39}$ with options (-g $\left.0-\mathrm{x} 0-\mathrm{m} 40-\mathrm{o} 15\right)$, and manually curated using gap5 v1.14. $8^{40}$. Smaller scaffolds were defined as residual haplotypes when they were entirely covered by $>90 \%$ within larger scaffolds and share half of the average coverage. These scaffolds were removed and Illumina paired end reads were remapped to check if the reads were successfully remapped to the larger scaffolds. Base and consensus correction was then performed with $8.8 \mathrm{~Gb}$ Illumina pair-end reads using ICORN2 $\mathrm{v} 0.95^{41}$ with five iterations. High molecular weight genomic DNA prepared from C. inopinata using CHEF Genomic DNA Plug Kits (BioRad) was mapped using the IRYS Mapping System (BioNano) and was used to improve the genome assembly to order and orientate sequence scaffolds, to measure the size of sequence gaps, and independently validate the sequence assembly, producing the v7 genome assembly.

Repeat analysis. Repeats within the assemblies were identified using the combined outputs of RepeatModeler (v1.0.4, http://www.repeatmasker.org/RepeatModeler. html) and TransposonPSI (v08222010, http://transposonpsi.sourceforge.net). UCLUST was used to cluster repeat sequences from RepeatModeler and TransposonPSI that had $\geq 80 \%$ identity, to generate consensus sequences for a nonredundant repeat library. RepeatMasker (v.3.2.8, http://www.repeatmasker.org) was then run (using the slow search option) with a custom repeat library for each species, to calculate the distribution of each repeat and its abundance in the genome. LTR retrotransposable elements were separately identified using a combination of LTR harvest/digest v1.5.8 $8^{42,43}$ and MGEScan v1.3.1 $1^{44}$. LTRharvest was used to identify potential candidates of LTRs in the genome (LTR lengths 100-1000bp, total length $1-10 \mathrm{kbp}, 85 \%$ minimum LTR similarity, TSD length 4-20bp, seed 30bp, looking for motif starting 'tg' and ending in 'ca'). LTRdigest was used to subsequently annotate the candidates with protein domains from Pfam and GyDB, as well as putative primer-binding sites and polypurine tracts. MGEscanLTR was also run on the genome to identify LTRs de novo with default parameters. The two results were processed using custom scripts (available from github.com/ syubukun/Sp34) to remove low confidence candidates by only keeping (1) candidates with full LTR retrotransposon domain sets (RT, protease, integrase, RNase $\mathrm{H}),(2)$ candidates with at least one protein domain $(e$-value $<1 \mathrm{e}-20)$ but not full, and (3) candidates with only LTR regions but has high similarity $(>95 \%)$ to LTRs from (1).

Gene findings and annotation. To predict protein-coding genes, Augustus (v. 3.0.1) ${ }^{45}$ was trained for C. inopinata based on a training set of 1500 nonoverlapping, non-homologous, and manually curated genes. A selection of gene models initially predicted by CEGMA v2.5 $5^{46}$ and Augustus C. elegans parameters, were curated in Artemis ${ }^{47}$ using aligned RNA-seq data and BLAST matches ${ }^{48}$ against the NCBI database. Based on RNA-seq alignments to the genome using Hisat2 v2.0.3 ${ }^{49}$ (parameters: -rna-strandness RF-min-intronlen 20 -max-intronlen 10,000), the bam2hints programme (part of the Augustus package) was used to create the intron hints, with minimum length set to $20 \mathrm{bp}$. The mapped RNAseq reads were also assembled into transcripts using Cufflinks v2.2.1 with options (-min-intron-length 15, -max-intron-length 300,000). The predicted exons in the resultant set of transcripts were used as the exonpart hints. The trained versions of Augustus were run using all the hints for that species as input. If Augustus predicted multiple, alternatively spliced transcripts for a gene, we only kept the transcript corresponding to the longest predicted protein to have a total of 20,976 gene models (v7.7 gene set). Gene structures in the Augustus predictions were refined by manual curation in Artemis, using RNA-seq mapping results and BLAST result against $C$. elegans proteins. In the manual curation processes, we targeted mainly on erroneously fused gene models and GPCR genes, which we found difficult to predict using a machine prediction. If a gene was manually curated, it replaced the original Augustus gene prediction in our final gene set and this process increased the gene numbers to 21,609 (v7.10 gene set).

Identification of gene families and gene family evolution analysis. To establish orthology relationships among Caenorhabditis species, non-redundant proteomes 
of eight Caenorhabditis species and an outgroup species Pristionchus pacificus (Supplementary Table 9) were obtained from WormBase (version WS252). OrthoFinder version $0.2 .841^{50}$ with default options was used to assess orthology with those proteins. We used CAFE (version 3$)^{51}$ to analyse gene family expansion and contraction under maximum likelihood framework. Gene family results from OrthoFinder and ultrametric phylogenetic tree calculated as described below were used as inputs under parameters " $-\mathrm{p} 0.01,-\mathrm{r} 1000$ ".

Species tree reconstruction. Amino acid sequences in each single-copy gene family were aligned using MAFFT version v7.22 $1^{52}$, poorly aligned regions were trimmed using GBlocks v0.91b ${ }^{53}$, and then the 1000 trimmed alignments (all sequences in 16 alignments were removed in the Gblocks trimming) were concatenated. The best-fitting substitution model for each protein alignment was identified using the RAxML option (-m PROTGAMMAAUTO). A maximumlikelihood phylogenetic tree was produced based on the concatenated alignment using RAxML v8.2.7 ${ }^{54}$ with 500 bootstrap replicates.

Divergence estimate. Neutral divergence between C. inopinata and C. elegans was estimated according to the Cutter's method ${ }^{2,19}$. To begin with the estimation, lineage-specific rate of synonymous (dS) and non-synonymous (dN) substitution for orthologous groups of genes between each of Caenorhabditis species in the phylogeny was calculated using Codeml in PAML (v4.9) ${ }^{55}$ with options (runmode $=0$, CodonFreq $=2$, model $=1$, fix_omega $=0$, omega $=0.01$ ). Lineage ages $(T)$ were inferred by applying median of synonymous-site divergence values and direct measures of the average per-site mutation rate in C. elegans $\left(\mu=9.0 \times 10^{-9}\right.$ mutations per generation $)^{56}$ to the equation from the neutral theory of molecular evolution $T=\mathrm{dS} / \mu$. Effective codon usage numbers $(\mathrm{Nc})$ was calculated by DAMBE (v6) ${ }^{57}$ and used to adjust the divergence values upward by transforming least-squares regression of branch-specific dS to the expected dS for the $N c$. Extreme values $(\mathrm{dN}>0.5, \mathrm{dS}>5$, and $\mathrm{dS}<0.0005)$ were removed before estimation to avoid biases from saturation of synonymous rate between related species and miss assignment of orthologous groups.

Functional annotation. Functional annotations were performed on the C. inopinata gene models (v7.10) based on multiple pieces of evidence including BLASTP search against UniProt (http://www.uniprot.org/), the latest version Pfam search (ver. 30.0) ${ }^{58}$ with HMMER3, InterProScan ${ }^{59}, \mathrm{dbCAN}^{60}$ for Carbohydrate-active enzymes (CAZymes) search, and orthology information with C. elegans from OrthoFinder results (for details, please see Supplementary note 3). Gene ontology (GO) terms of the three possible types (molecular function, cellular component, and biological process) were assigned to genes by transferring GO terms from the C. elegans orthologues. Only manually curated GO annotations were downloaded from the GO Consortium website and transferred to the C. inopinata. Additional GO terms were identified using Blast2Go (v2) ${ }^{61}$ with BLAST search against NCBI nr database and the InterProScan ${ }^{59}$ results.

GPCR analyses. We manually curated 7TM-GPCR genes in the C. inopinata genome based on TBLATN search using C. elegans serpentines as queries. To see detailed relationships of Caenorhabditis, phylogenetic trees of genes in each serpentine family from C. inopinata, C. elegans, and C. briggsae were constructed using Mafft v.7.221 ${ }^{52}$ and FastTree 2.1.8

Synteny analysis. Using the three highly contiguous species, $C$. inopinata, $C$. elegans, and C. briggsae were used for synteny analysis. Synteny was detected by DAGchainer $(\mathrm{r} 02062008)^{63}$ using the orthology of protein-coding genes assigned as described above. Synteny-linking plots were generated using segments and polygon functions in R (version 3.2.4) (for three species) and Circos (v0.67-7) ${ }^{64}$ (for C. elegans and C. inopinata). Bedtools (v2.17.0) ${ }^{65}$ was used to compute size of gene, intergenic space per synteny block and to obtain non-synteny regions along each chromosome.

Transgenesis by microinjection. The germline transformation in C. inopinata was performed by microinjection according to the protocol commonly used for $C$. elegans $^{66}$. Worms for microinjection were fed with E. coli OP50-1 or HT115(DE3) (which enhances germline proliferation compared with OP50-1). The plasmid mixture was microinjected into the proximal gonads of $C$. inopinata adult females and recovered worms were cultured with adult males. Transgenic progeny was screened under the dissecting microscope. Transgenic marker plasmids commonly used in C. elegans were used to construct transgenic C. inopinata. The composition of the injected plasmid mixture was the following: $40 \mathrm{ng} / \mu \mathrm{L}$ pRF4 (dominant rol-6 mutant that causes dominant Rol phenotype) ${ }^{66}, 35 \mathrm{ng} / \mu \mathrm{L}$ pTG96 (SUR-5::GFP; nuclear GFP in all cells) ${ }^{67}, 40 \mathrm{ng} / \mu \mathrm{L}$ Podr-1::GFP (GFP in AWC neurons) ${ }^{68}, 40 \mathrm{ng} /$ $\mu \mathrm{L}$ pCFJ68 (Punc-122::GFP; GFP in coelomocytes, Addgene plasmid \#19325, a gift from Erik Jorgensen) ${ }^{69}, 40 \mathrm{ng} / \mu \mathrm{L}$ Punc-122::RFP (RFP in coelomocytes, Addgene plasmid \#8938, a gift from Piali Sengupta) ${ }^{70}$, and $40 \mathrm{ng} / \mu \mathrm{L}$ pCFJ104 (Pmyo-3: mCherry; mCherry in body wall muscles, Addgene plasmid \#19328, a gift from Erik Jorgensen ${ }^{69}$. Multiple stable transgenic lines were obtained, and one of them was used for the following RNAi experiment.
Immunofluorescence using antibodies against $C$. elegans proteins. C. inopinata embryos were immunostained as described for $C$. elegans ${ }^{71}$. Briefly, embryos were collected by cutting gravid female adults, permeabilized by freeze-cracking, and fixed with methanol-acetone. Polyclonal rat anti-Ce-PGL-3 OB0706 (1:10,000, MBL; generated using His-tagged Ce-PGL-3 as the antigen $)^{15}$ or rat polyclonal anti-Ce-TBG-1 (1:100, MBL; generated using His-tagged Ce-TBG-1 as the antigen $)^{14}$ were used as primary antibodies. Goat Alexa Fluor 488 or 568 anti-rat IgG (1:200) (Thermo Fisher Scientific, Waltham, MA, USA) were used as secondary antibodies. DNA was stained with $10 \mathrm{ng} / \mu \mathrm{L}$ DAPI (4',6-diamidino-2phenylindole) (Dojindo Laboratories, Kumamoto, Japan).

Feeding and soaking RNAi. Feeding RNAi in C. inopinata was performed according to the protocol established for C. elegans ${ }^{72}$. To knock-down GFP in the transgenic lines, the coding region of the GFP transgene was cloned into the feeding plasmid vector L4440 that induces dsRNA expression in E. coli. To knockdown the unc-22 orthologue in C. inopinata, genomic fragment of the C. inopinata unc-22 gene (Cin-unc-22) was cloned into L4440. Fifteen adults C. inopinata females carrying eggs were placed onto feeding plates seeded with the feeding bacteria (E. coli HT115(DE3) transformed with a feeding RNAi vector), and phenotypes in the next generation were analysed on the 5th day. Soaking RNAi in C. inopinata was performed according to the protocol established for C. elegans $s^{73}$ To knock-down the $t b g-1$ orthologue in $C$. inopinata, the genomic sequences corresponding to the C. elegans tbg-1 gene was PCR-amplified using the primers with T7 RNA polymerase primers, and used the amplified fragments as templates for dsRNA synthesis in vitro. As a control, dsRNA for GFP were used. L4 males and females of C. inopinata were soaked in the dsRNA solution $(\sim 1 \mathrm{mg} / \mathrm{mL})$ for 24 $h$, and the phenotypes of the progeny of recovered worms were analysed.

Florescence microscopy. Differential interference contrast (DIC) and fluorescent images were obtained using Zeiss Axioplan 2 imaging microscope (Carl Zeiss, Jena, Germany) with $10 \times / 0.30$ NA Plan-NEOFLUAR or $\times 63 / 1.20$ water Korr UV-VIS-IR objective lenses with Orca-R2 12-bit_16-bit cooled CCD camera (Hamamatsu Photonics, Hamamatsu, Japan), under the control of Metamorph software (Molecular Devices, Sunnyvale, CA, USA). Obtained images were processed by Image J/Fiji software (National Institutes of Health, Bethesda, MD, USA).

Methods for ascaroside analysis. Preparation of metabolite extracts: $C$. inopinata was maintained at $25^{\circ} \mathrm{C}$ on NGM agar plates, made with Bacto agar (BD Biosciences) and seeded with E. coli OP50 grown overnight. Mixed stage C. inopinata worms were rinsed from plates in M9 buffer and allowed to settle to the bottom of a $15 \mathrm{~mL}$ conical tube. After settling, the supernatant was removed and worms were resuspended in fresh M9 buffer. This rinsing procedure was repeated for a total of four times. Clean worms were then placed in $100 \mathrm{~mL}$ of M9 buffer at a concentration of 1 worm per microliter and incubated on a rotary shaker at $20^{\circ} \mathrm{C}$ for $6 \mathrm{~h}$. The cultures were then centrifuged and the resulting supernatant and worm pellet were snap-frozen in liquid nitrogen and stored at $-20^{\circ} \mathrm{C}$. The frozen samples were lyophilised and extracted with 95:5 ethanol:water for $12 \mathrm{~h}$. The extract was dried in vacuo, resuspended in methanol and analysed by LC/MS. LC/ MS analysis was performed on a Dionex 3000 UPLC coupled to a Thermo Q Exactive high-resolution mass spectrometer. Metabolites were separated using a water-acetonitrile gradient on Agilent Zorbax Eclipse XDB-C18 column, $150 \times 2.1$ $\mathrm{mm}$, particle size $1.8 \mu \mathrm{m}$, maintained at $40^{\circ} \mathrm{C}$. Solvent A: $0.1 \%$ formic acid in water, solvent B: $0.1 \%$ formic acid in acetonitrile. A/B gradient started at $5 \% \mathrm{~B}$ at $1.5 \mathrm{~min}$ after injection and increased linearly to $100 \%$ B at $12.5 \mathrm{~min}$. Ascarosides were detected as $[\mathrm{M}-\mathrm{H}]$-ions in the negative ionisation mode. Metabolites were identified based on their high-resolution masses $(<1 \mathrm{ppm})$, fragmentation spectra, and comparison of retention times with those of synthetic standards and known metabolites.

Retention assay protocol: Standard $5 \mathrm{~cm}$ agar plates (made with standard Nematode Growth Medium) were seeded with $100 \mu \mathrm{L}$ of OP50 E. coli. The $16 \mathrm{~mm}$ bacterial lawn was grown overnight at $20^{\circ} \mathrm{C}$ before being used in trials. Worms were grown at $20^{\circ} \mathrm{C}$ on live OP50 E. coli on standard NGM agar plates. 9-10-dayold well-fed males were picked from plates containing both males and females onto a new seeded plate the day before experiments (males had no contact with female worms overnight). Using a transparent template to guide spot placement, two $4 \mathrm{~mm}$ spots $(1 \mu \mathrm{L})$ of the relevant solutions were placed on opposite sides of the bacterial lawn and left to dry for several minutes before nematode placement. Recording began immediately upon nematode placement ${ }^{74}$. A drop of the control was placed on one side of the lawn and a drop of the experimental cue was placed on the other side of the lawn. The location of cues was switched throughout the trials, between left/right to minimise bias. A mixture containing the appropriate amounts of the best performing ascarosides (100 fmol Ascr\#1, 1 fmol Ascr\#3, and 1 pmol Ascr\#10) was also tested. 9-10-day-old adult males were isolated the day before being used in trials. All worms were placed at the midpoint between the foci of both scoring regions. Trials were recorded for $20 \mathrm{~min}$ and frames were collected at one frame per second. The first $15 \mathrm{~min}$ of each video was used for analysis. Results were averaged from 3 to 6 trials. Ten worms were used per assay. We used automated software to compare worm occupancy in each scoring region over time, then adapted the Chemotaxis Index to score preference or avoidance to each 
ascaroside. Student's two-tailed $T$-test was used to compare the Chemotaxis Index for each treatment to that of a water control (two spots of water on either side of the lawn). ${ }^{*} p<0.05,{ }^{* *} p<0.01,{ }^{* *} p<0.001$.

Data availability. All sequence data from the C. elegans sibling genome project have been deposited at DDBJ/ENA/GenBank under BioProject accession PRJDB5687. Taxonomic descriptions were deposited at Zoobank. Nematode specimen and strains are available from T. Kikuchi or N. Kanzaki on request. All relevant data are available from the corresponding authors upon reasonable request.

Received: 8 December 2017 Accepted: 19 July 2018

Published online: 10 August 2018

\section{References}

1. Antoshechkin, I. \& Sternberg, P. W. The versatile worm: genetic and genomic resources for Caenorhabditis elegans research. Nat. Rev. Genet. 8, 518-532 (2007).

2. Cutter, A. D. Divergence times in Caenorhabditis and Drosophila inferred from direct estimates of the neutral mutation rate. Mol. Biol. Evol. 25, 778-786 (2008).

3. Kiontke, K. C. et al. A phylogeny and molecular barcodes for Caenorhabditis, with numerous new species from rotting fruits. BMC Evol. Biol. 11, 339 (2011).

4. Felix, M. A., Braendle, C. \& Cutter, A. D. A streamlined system for species diagnosis in Caenorhabditis (Nematoda: Rhabditidae) with name designations for 15 distinct biological species. PLoS ONE 9, e94723 (2014).

5. Conchou, L., Cabioch, L., Rodriguez, L. J. \& Kjellberg, F. Daily rhythm of mutualistic pollinator activity and scent emission in Ficus septica: ecological differentiation between co-occurring pollinators and potential consequences for chemical communication and facilitation of host speciation. PLOS ONE 9, e103581 (2014)

6. Reinke, S. N., Hu, X., Sykes, B. D. \& Lemire, B. D. Caenorhabditis elegans diet significantly affects metabolic profile, mitochondrial DNA levels, lifespan and brood size. Mol. Genet. Metab. 100, 274-282 (2010).

7. Pang, S. \& Curran, S. P. Adaptive capacity to bacterial diet modulates aging in C. elegans. Cell. Metab. 19, 221-231 (2014).

8. Chi, C. et al. Nucleotide levels regulate germline proliferation through modulating GLP-1/Notch signaling in C. elegans. Genes Dev. 30, 307-320 (2016).

9. Coghlan, A. Nematode genome evolution. WormBook, 1-15, https://doi.org/ 10.1895/wormbook.1.15.1 (2005).

10. The-Caenorhabditis-elegans-Sequencing-Consortium. Genome sequence of the nematode C. elegans: a platform for investigating biology. Science 282, 2012-2018 (1998).

11. LaMunyon, C. W. \& Ward, S. Increased competitiveness of nematode sperm bearing the male X chromosome. Proc. Natl Acad. Sci. USA 94, 185-189 (1997).

12. Hamilton, W. D. Extraordinary sex ratios. A sex-ratio theory for sex linkage and inbreeding has new implications in cytogenetics and entomology. Science 156, 477-488 (1967).

13. Nayak, S., Goree, J. \& Schedl, T. fog-2 and the evolution of self-fertile hermaphroditism in Caenorhabditis. PLoS Biol. 3, e6 (2005).

14. Toya, M., Terasawa, M., Nagata, K., Iida, Y. \& Sugimoto, A. A kinaseindependent role for Aurora A in the assembly of mitotic spindle microtubules in Caenorhabditis elegans embryos. Nat. Cell Biol. 13, 708-714 (2011).

15. Hanazawa, M., Yonetani, M. \& Sugimoto, A. PGL proteins self associate and bind RNPs to mediate germ granule assembly in C. elegans. J. Cell Biol. 192, 929-937 (2011).

16. Robertson, H. M. \& Thomas, J. H. The putative chemoreceptor families of $C$. elegans. WormBook, 1-12, https://doi.org/10.1895/wormbook.1.66.1 (2006).

17. Ludewig, A. H. \& Schroeder, F. C. Ascaroside signaling in C. elegans. WormBook, 1-22, https://doi.org/10.1895/wormbook.1.155.1 (2013).

18. Fierst, J. L. et al. Reproductive mode and the evolution of genome size and structure in Caenorhabditis nematodes. PLoS Genet. 11, e1005323 (2015).

19. Thomas, C. G. et al. Full-genome evolutionary histories of selfing, splitting, and selection in Caenorhabditis. Genome Res. 25, 667-678 (2015).

20. Yin, D. et al. Rapid genome shrinkage in a self-fertile nematode reveals sperm competition proteins. Science 359, 55-61 (2018).

21. Wang, J., Chen, P. J., Wang, G. J. \& Keller, L. Chromosome size differences may affect meiosis and genome size. Science 329, 293 (2010).

22. Le, T. S. et al. Non-Mendelian assortment of homologous autosomes of different sizes in males is the ancestral state in the Caenorhabditis lineage. Sci. Rep. 7, 12819 (2017).
23. Fischer, S. E. J. et al. The ERI-6/7 Helicase acts at the first stage of an siRNA amplification pathway that targets recent gene duplications. PLoS Genet. 7 , e1002369 (2011).

24. Vasale, J. J. et al. Sequential rounds of RNA-dependentRNA transcription drive endogenous small-RNA biogenesis in the ERGO-1/Argonaute pathway. Proc. Natl Acad. Sci. USA 107, 3582-3587 (2010)

25. Nuez, I. \& Felix, M. A. Evolution of susceptibility to ingested double-stranded RNAs in Caenorhabditis nematodes. PLoS ONE 7, e29811 (2012).

26. Billi, A. C., Fischer, S. E. \& Kim, J. K. Endogenous RNAi pathways in C. elegans. WormBook, 1-49, https://doi.org/10.1895/wormbook.1.170.1 (2014).

27. Stein, L. D. et al. The genome sequence of Caenorhabditis briggsae: a platform for comparative genomics. PLoS Biol. 1, E45 (2003).

28. Cutter, A. D., Dey, A. \& Murray, R. L. Evolution of the Caenorhabditis elegans genome. Mol. Biol. Evol. 26, 1199-1234 (2009).

29. Ross, J. A. et al. Caenorhabditis briggsae recombinant inbred line genotypes reveal inter-strain incompatibility and the evolution of recombination. PLoS Genet. 7, e1002174 (2011)

30. Hooper, D. Handling, Fixing, Staining and Mounting Nematodes. Technical Bulletin, 39-54 (Ministry of Agriculture, Fisheries and Food, 1970).

31. Bolger, A. M., Lohse, M. \& Usadel, B. Trimmomatic: a flexible trimmer for Illumina sequence data. Bioinformatics 30, 2114-2120 (2014).

32. Leggett, R. M., Clavijo, B. J., Clissold, L., Clark, M. D. \& Caccamo, M. NextClip: an analysis and read preparation tool for Nextera Long Mate Pair libraries. Bioinformatics 30, 566-568 (2014).

33. Kajitani, R. et al. Efficient de novo assembly of highly heterozygous genomes from whole-genome shotgun short reads. Genome Res. 24, 1384-1395 (2014).

34. Chin, C.-S. et al. Phased diploid genome assembly with single-molecule realtime sequencing. Nat. Methods 13, 1050-1054 (2016).

35. Koren, S., Walenz, B. P., Berlin, K., Miller, J. R. \& Phillippy, A. M. Canu: scalable and accurate long-read assembly via adaptive $\mathrm{k}$-mer weighting and repeat separation. bioRxiv. https://doi.org/10.1101/071282 (2016).

36. Chin, C. S. et al. Nonhybrid, finished microbial genome assemblies from longread SMRT sequencing data. Nat. Methods 10, 563-569 (2013).

37. Wences, A. H. \& Schatz, M. C. Metassembler: merging and optimizing de novo genome assemblies. Genome Biol. 16, 207 (2015).

38. Huang, S. et al. HaploMerger: reconstructing allelic relationships for polymorphic diploid genome assemblies. Genome Res. 22, 1581-1588 (2012).

39. Boetzer, M. \& Pirovano, W. SSPACE-LongRead: scaffolding bacterial draft genomes using long read sequence information. BMC Bioinform. 15, 211 (2014).

40. Bonfield, J. K. \& Whitwham, A. Gap5-editing the billion fragment sequence assembly. Bioinformatics 26, 1699-1703 (2010).

41. Otto, T. D., Sanders, M., Berriman, M. \& Newbold, C. Iterative correction of reference nucleotides (iCORN) using second generation sequencing technology. Bioinformatics 26, 1704-1707 (2010).

42. Steinbiss, S., Willhoeft, U., Gremme, G. \& Kurtz, S. Fine-grained annotation and classification of de novo predicted LTR retrotransposons. Nucleic Acids Res. 37, 7002-7013 (2009).

43. Ellinghaus, D., Kurtz, S. \& Willhoeft, U. LTRharvest, an efficient and flexible software for de novo detection of LTR retrotransposons. BMC Bioinform. 9, 18 (2008).

44. Lee, H. et al. MGEScan: a Galaxy-based system for identifying retrotransposons in genomes. Bioinformatics 32, 2502-2504 (2016).

45. Stanke, M., Steinkamp, R., Waack, S. \& Morgenstern, B. AUGUSTUS: a web server for gene finding in eukaryotes. Nucleic Acids Res. 32, W309-W312 (2004).

46. Parra, G., Bradnam, K., Ning, Z., Keane, T. \& Korf, I. Assessing the gene space in draft genomes. Nucleic Acids Res. 37, 289-297 (2009).

47. Rutherford, K. et al. Artemis: sequence visualization and annotation. Bioinformatics 16, 944-945 (2000).

48. Altschul, S. F. et al. Gapped BLAST and PSI-BLAST: a new generation of protein database search programs. Nucleic Acids Res. 25, 3389-3402 (1997).

49. Kim, D., Langmead, B. \& Salzberg, S. L. HISAT: a fast spliced aligner with low memory requirements. Nat. Methods 12, 357-360 (2015).

50. Emms, D. M. \& Kelly, S. OrthoFinder: solving fundamental biases in whole genome comparisons dramatically improves orthogroup inference accuracy. Genome Biol. 16, 157 (2015).

51. Han, M. V., Thomas, G. W., Lugo-Martinez, J. \& Hahn, M. W. Estimating gene gain and loss rates in the presence of error in genome assembly and annotation using CAFE 3. Mol. Biol. Evol. 30, 1987-1997 (2013).

52. Katoh, K., Misawa, K., Kuma, K. \& Miyata, T. MAFFT: a novel method for rapid multiple sequence alignment based on fast Fourier transform. Nucleic Acids Res. 30, 3059-3066 (2002).

53. Talavera, G. \& Castresana, J. Improvement of phylogenies after removing divergent and ambiguously aligned blocks from protein sequence alignments. Syst. Biol. 56, 564-577 (2007). 
54. Stamatakis, A. RAxML-VI-HPC: maximum likelihood-based phylogenetic analyses with thousands of taxa and mixed models. Bioinformatics 22, 2688-2690 (2006).

55. Yang, Z. PAML 4: phylogenetic analysis by maximum likelihood. Mol. Biol. Evol. 24, 1586-1591 (2007)

56. Denver, D. R., Morris, K., Lynch, M. \& Thomas, W. K. High mutation rate and predominance of insertions in the Caenorhabditis elegans nuclear genome. Nature 430, 679-682 (2004).

57. Sun, X., Yang, Q. \& Xia, X. An improved implementation of effective number of codons (nc). Mol. Biol. Evol. 30, 191-196 (2013).

58. Finn, R. D. et al. Pfam: the protein families database. Nucleic Acids Res. 42 D222-D230 (2014).

59. Quevillon, E. et al. InterProScan: protein domains identifier. Nucleic Acids Res. 33, W116-W120 (2005).

60. Yin, Y. et al. dbCAN: a web resource for automated carbohydrate-active enzyme annotation. Nucleic Acids Res. 40, W445-W451 (2012)

61. Conesa, A. et al. Blast2GO: a universal tool for annotation, visualization and analysis in functional genomics research. Bioinformatics 21, 3674-3676 (2005).

62. Price, M. N., Dehal, P. S. \& Arkin, A. P. FastTree 2-approximately maximum-likelihood trees for large alignments. PLoS ONE 5, e9490 (2010).

63. Haas, B. J., Delcher, A. L., Wortman, J. R. \& Salzberg, S. L. DAGchainer: a tool for mining segmental genome duplications and synteny. Bioinformatics $\mathbf{2 0}$, 3643-3646 (2004).

64. Krzywinski, M. et al. Circos: an information aesthetic for comparative genomics. Genome Res. 19, 1639-1645 (2009).

65. Quinlan, A. R. \& Hall, I. M. BEDTools: a flexible suite of utilities for comparing genomic features. Bioinformatics 26, 841-842 (2010).

66. Mello, C. C., Kramer, J. M., Stinchcomb, D. \& Ambros, V. Efficient gene transfer in C. elegans: extrachromosomal maintenance and integration of transforming sequences. EMBO J. 10, 3959-3970 (1991).

67. Yochem, J., Gu, T. \& Han, M. A new marker for mosaic analysis in Caenorhabditis elegans indicates a fusion between hyp6 and hyp7, two major components of the hypodermis. Genetics 149, 1323-1334 (1998).

68. L'Etoile, N. D. \& Bargmann, C. I. Olfaction and odor discrimination are mediated by the C. elegans guanylyl cyclase ODR-1. Neuron 25, 575-586 (2000).

69. Frokjaer-Jensen, C. et al. Single-copy insertion of transgenes in Caenorhabditis elegans. Nat. Genet. 40, 1375-1383 (2008).

70. Miyabayashi, T., Palfreyman, M. T., Sluder, A. E., Slack, F. \& Sengupta, P. Expression and function of members of a divergent nuclear receptor family in Caenorhabditis elegans. Dev. Biol. 215, 314-331 (1999).

71. Takeda, K., Watanabe, C., Qadota, H., Hanazawa, M. \& Sugimoto, A. Efficient production of monoclonal antibodies recognizing specific structures in Caenorhabditis elegans embryos using an antigen subtraction method. Genes Cells 13, 653-665 (2008).

72. Timmons, L. \& Fire, A. Specific interference by ingested dsRNA. Nature 395, 854 (1998).

73. Maeda, I., Kohara, Y., Yamamoto, M. \& Sugimoto, A. Large-scale analysis of gene function in Caenorhabditis elegans by high-throughput RNAi. Curr. Biol. 11, 171-176 (2001)

74. Chai, C. M., Cronin, C. J. \& Sternberg, P. W. Automated analysis of a nematode population-based chemosensory preference assay. J. Vis. Exp. 125, e55963 (2017).
75. Llorens, C. et al. The Gypsy Database (GyDB) of mobile genetic elements: release 2.0. Nucleic Acids Res. 39, D70-D74 (2010).

\section{Acknowledgements}

This work was supported by Japan Society for the Promotion of Science (JSPS) KAKENHI Grant Numbers 26292178, 16H04722, and 15K14503. V.L.H. was supported by JSPS International Research Fellowship, A.T., N.H., S.R.D., and M.B. by the core funding at the Wellcome Trust Sanger Institute (grant no. 206194), P.W.S. by Howard Hughes Medical Institute. Genome data analyses were partly performed using the DDBJ supercomputer system. We thank Akemi Yoshida, Mark Bligh, Mohammad-Masum Billah, Tanzila Afrin, Aya Adachi, Shakhinur Islam Mondal, Arzuba Akter, Haruhiko Maruyama, Tzu-hao Kuo, Karen Brooks, Michelle Smith for assistance and comments.

\section{Author contributions}

T.K. and N.K. conceived the study. P.W.S., A.S., F.S., I.J.T., N.K., and T.K. contributed to study design. V.L.H., A.S., I.J.T., P.W.S., N.K., and T.K. wrote the manuscript with inputs from others. N.K., R.T., K.T., S.N., R.K., H.K., H.T., C.C., A.A., O.P., and J.W. conducted experiments. N.K. and G.C.W. provided the strains. I.J.T., A.T., S.R.D., N.H., T.K., and M.B. completed genome assembly. V.L.H., Y.M., K.M., D.L., I.J.T., and T.K. analysed genome data.

\section{Additional information}

Supplementary Information accompanies this paper at https://doi.org/10.1038/s41467 018-05712-5.

Competing interests: The authors declare no competing interests.

Reprints and permission information is available online at http://npg.nature.com/ reprintsandpermissions/

Publisher's note: Springer Nature remains neutral with regard to jurisdictional claims in published maps and institutional affiliations.

\begin{abstract}
Open Access This article is licensed under a Creative Commons BY Attribution 4.0 International License, which permits use, sharing,
adaptation, distribution and reproduction in any medium or format, as long as you give appropriate credit to the original author(s) and the source, provide a link to the Creative Commons license, and indicate if changes were made. The images or other third party material in this article are included in the article's Creative Commons license, unless indicated otherwise in a credit line to the material. If material is not included in the article's Creative Commons license and your intended use is not permitted by statutory regulation or exceeds the permitted use, you will need to obtain permission directly from the copyright holder. To view a copy of this license, visit http://creativecommons.org/ licenses/by/4.0/.
\end{abstract}

(C) The Author(s) 2018 